\title{
A renormalization approach to irrational rotations
}

\author{
Claudio Bonanno* Stefano Isola ${ }^{\dagger}$
}

October 31, 2018

\begin{abstract}
We introduce a renormalization procedure which allows us to study in a unified and concise way different properties of the irrational rotations on the unit circle $\beta \mapsto\{\alpha+\beta\}, \alpha \in \mathbb{R} \backslash \mathbb{Q}$. In particular we obtain sharp results for the diffusion of the walk on $\mathbb{Z}$ generated by the location of points of the sequence $\{n \alpha+\beta\}$ on a binary partition of the unit interval. Finally we give some applications of our method.
\end{abstract}

2000 Mathematics Subject Classification: 37E05, 37A25, 37A45

Key words and phrases: Irrational rotations, continued fractions, renormalization, diffusion.

\section{Introduction}

Irrational rotations on the unit circle $S^{1} \cong[0,1] /(0=1)$ are isometric transformations defined by

$$
[0,1) \ni \beta \mapsto\{\alpha+\beta\} \in[0,1)
$$

where $\alpha \in \mathbb{R} \backslash \mathbb{Q}$ is the angle of rotation and $\{\cdot\}$ denotes the fractional part of a real number. It is well known that the Lebesgue measure on the unit interval is the unique (and thus ergodic) invariant probability measure for these transformations. However, some ergodic properties of the rotations, such as recurrence rates, waiting times and some limit laws, are known to depend on the arithmetic properties of the rotation angle $\alpha$ (see [B2], $[\mathrm{CD}], \mathrm{CF}],[\mathrm{K}], \mathrm{KS}])$. These, in turn, are encoded in its continued fraction

\footnotetext{
*Dipartimento di Matematica Applicata, Università di Pisa, via F. Buonarroti 1/c, 56127 Pisa (Italy), email: <bonanno@mail.dm.unipi.it>;

${ }^{\dagger}$ Dipartimento di Matematica e Informatica, Università di Camerino, via Madonna delle Carceri, 62032 Camerino (Italy), e-mail: <stefano.isola@unicam.it>
} 
expansion and it is starting from this expansion that we introduce, borrowing it from dynamical systems, a renormalization procedure which allows us to study several relevant properties of the orbit $(\{n \alpha+\beta\})$. The possibility of studying all these properties through the same approach is one of the main motivations of this paper. Indeed, the paper is self-contained and we obtain sharp results using efficient and concise arguments based solely on the renormalization procedure.

A renormalization approach to circle maps was introduced in $\mathrm{L}$, and used for example in $[\mathrm{CF}$ to estimate limit laws of entrance times for irrational rotations. We remark that the renormalization procedure we use is quite different from the one in $[\mathrm{L}]$, both in the construction and in the spirit. Moreover, we think that our approach can be used successfully to give sharp estimates on some more ergodic properties and limit laws than those considered in this paper (see [B]).

In this paper, we study the distribution properties of the sequences $x_{n}:=$ $n \alpha+\beta, n=0,1,2, \ldots$. We recall that a sequence $\left(y_{n}\right)$ of real numbers is said to be uniformly distributed modulo 1 , if for any real number $0<\gamma \leq 1$ we have

$$
\lim _{n \rightarrow \infty} \frac{1}{n} \sum_{r=0}^{n-1} \chi_{[0, \gamma)}\left(\left\{y_{r}\right\}\right)=\gamma
$$

where $\chi_{A}$ denotes the indicator function of the set $A$.

An interesting characterisation of the distribution properties of a sequence $\left(y_{n}\right)$ can be obtained as follows: take the partition of the unit interval given by $\left\{\left[0, \frac{1}{2}\right),\left[\frac{1}{2}, 1\right)\right\}$ and construct the walk on $\mathbb{Z}$ which starts at the origin at time 0 and at time $n+1$ moves one step to the right if $\left\{y_{n}\right\} \in\left[0, \frac{1}{2}\right)$, one step to the left otherwise. After $n+1$ steps, the position of the walker is given by

$$
\begin{gathered}
S_{n}=\sum_{r=0}^{n} s\left(\left\{y_{r}\right\}\right) \\
s(y):=2 \chi_{\left[0, \frac{1}{2}\right)}(\{y\})-1 .
\end{gathered}
$$

Clearly, if $\left(y_{n}\right)$ is uniformly distributed modulo 1 then $\left|S_{n}\right|=o(n)$ as $n \rightarrow \infty$ and the better is the uniform distribution of $\left(y_{n}\right)$ the slower is the diffusion of the walk. In particular, for the ideal distribution for which $\frac{1}{n} \sum_{r=0}^{n-1} \chi_{[0, \gamma)}\left(\left\{y_{r}\right\}\right)=\gamma$ for all $n$ we have $S_{n}=0$ for all $n$.

In [I] the growth of the quantity $S_{n}$ has been studied for the sequence $\left(x_{n}\right)=(n \alpha+\beta)$ in the $L^{\infty}$ and $L^{2}$ norms. In this paper we give an explicit 
formula for $S_{n}$ and obtain as a corollary growth estimates for $\beta=0$, and a sharp result for the $L^{\infty}$ norm (see Corollary 4.5), improving results in [I]. For the sequence $x_{n}:=n \alpha+\beta$, with $\alpha$ irrational and $\beta \in(0,1)$, the indicator $S_{n}$ will be denoted by $S_{n}(\alpha, \beta)\left(S_{n}(\alpha)\right.$ if $\left.\beta=0\right)$ to stress its dependence on the arithmetical properties of the number $\alpha$.

The paper is organized as follows. Notations and the main ideas are settled in Section 2 which includes a preliminary analysis of several quantities that are needed for the renormalization procedure. In particular, we study how the different points of the sequence $(n \alpha)$ are organized according to their integer part. This depends on $a_{1}$, the first partial quotient in the continued fraction expansion of $\alpha$, and in particular by its parity. The main result of this section is Theorem 2.3 .

In Section 3 we give an iterative method to obtain an explicit expression for $S_{n}(\alpha)$ only in terms of the coefficients of the continued fraction of $\alpha$. This method is then used to obtain sharp estimates for $S_{n}(\alpha)$ that have significantly different expressions according to whether $a_{1}$ is even or odd. The main results are the algorithm described in Proposition 3.1 and its consequences described in Theorem 3.2 as well as in the subsequent examples. We point out that we obtain results also for the minimum values of $S_{n}(\alpha)$, with the aim of giving hints on the returns to zero (this point is analysed in [B]).

Next, we consider the general case of the sums $S_{n}(\alpha, \beta)$. Again we obtain an explicit expression for these sums (Theorem 4.4) in terms of the coefficient of the continued fraction of $\alpha$ and of the coefficient of the expansion of $\beta$ introduced in Proposition 2.4. In particular we obtain as a corollary a sharp estimate on the $L^{\infty}$ norm of $S_{n}(\alpha, \beta)$ (see Corollary 4.5).

Finally, we give some applications of our approach to the Birkhoff Ergodic Theorem and to the discrepancy (see (4.2)) of the sequence $(n \alpha)$. It is surprising that these results easily follow from our renormalization approach.

\section{Continued fractions and return sequences}

For a given number $\alpha \in(0,1)$ let us consider its expansion in continued fraction $[\mathrm{PF}]$

$$
\alpha=\frac{1}{a_{1}+\frac{1}{a_{2}+\frac{1}{a_{3}+\cdots}}}
$$


which we denote by $\alpha=\left[a_{1}, a_{2}, a_{3}, \ldots\right]$. The partial quotients $a_{h}$ are positive integers and the expansion terminates if and only if $\alpha$ is rational. If $\alpha$ is irrational its "fast" rational approximants are the numbers

$$
\frac{p_{n}}{q_{n}}:=\left[a_{1}, \ldots, a_{n}\right]
$$

which can be also be defined recursively by

$$
\frac{p_{0}}{q_{0}}=\frac{0}{1}, \quad \frac{p_{1}}{q_{1}}=\frac{1}{a_{1}} \quad \text { and } \quad \frac{p_{n+1}}{q_{n+1}}=\frac{a_{n+1} p_{n}+p_{n-1}}{a_{n+1} q_{n}+q_{n-1}}, \quad n \geq 1 .
$$

In the following we shall consider also the positive numbers

$$
f_{n}:=(-1)^{n}\left(q_{n} \alpha-p_{n}\right), \quad n \geq 0 .
$$

To a given $\alpha \in(0,1)$ we associate the rotation $T_{\alpha}: X \rightarrow X$ of the unit circle $X=[0,1] /(0=1)$ given by

$$
T_{\alpha}(\beta):=\{\alpha+\beta\} .
$$

One easily checks that the numbers $f_{n}$ determine the successive closest returns of the orbit of a point $x$ to the point itself (thus forming a monotonically decreasing sequence). Indeed, for all $n \geq 1$ and for all $\beta \in X$, it holds

$$
q_{n}=\min \left\{r>q_{n-1}:\left|x-T_{\alpha}^{r}(\beta)\right|<\left|\beta-T_{\alpha}^{q_{n-1}}(\beta)\right|\right\}
$$

and

$$
f_{n}=\left|\beta-T_{\alpha}^{q_{n}}(\beta)\right| .
$$

The first numbers $f_{n}$ are given by $f_{0}=\alpha, f_{1}=1-a_{1} \alpha, f_{2}=f_{0}-a_{2} f_{1}$ and more generally they satisfy the recursion

$$
f_{n+1}=f_{n-1}-a_{n+1} f_{n}, \quad n \geq 1 .
$$

Conversely, once the $f_{n}$ are known the partial quotients can be obtained as:

$$
a_{n+1}=\max \left\{h \geq 1: h f_{n}<f_{n-1}\right\}, \quad n \geq 0,
$$

with the position $f_{-1}=1$. This yields in particular

$$
\begin{gathered}
a_{1}=\max \{h \geq 1: h \alpha<1\} \\
a_{2}=\max \left\{h \geq 1: h\left(1-a_{1} \alpha\right)<\alpha\right\} .
\end{gathered}
$$


Note that $\lfloor r \alpha\rfloor=0$ for all $0 \leq r \leq a_{1}$ and $\left\lfloor\left(a_{1}+1\right) \alpha\right\rfloor=1$. In the sequel we shall study the behaviour of the sum $S_{n}(\alpha)$ by looking at the values of $s(r \alpha)$ with $\lfloor r \alpha\rfloor$ constant. To this end we introduce the following quantities. Set

$$
r_{k}:=\min \{r \geq 0:\lfloor r \alpha\rfloor=k\}, \quad k \geq 0 .
$$

In terms of $T_{\alpha}$ this is the least number of iterates of 0 needed to make $k$ "turns" of the circle $X$. Set moreover

$$
t_{k}:=\#\{r \geq 0:\lfloor r \alpha\rfloor=k\}, \quad k \geq 0,
$$

which is the number of $T_{\alpha}$-iterates of 0 which are all lying "within the same circle" after having turned the circle $k$ times.

One sees that $t_{0}=a_{1}+1$, and it is not difficult to realize that for all $k \geq 1$, $t_{k}$ is equal to either $a_{1}$ or $a_{1}+1$. More precisely we have

Lemma 2.1. $t_{k}$ is either equal to $a_{1}+1$ or $a_{1}$, according to whether $\left\{r_{k} \alpha\right\}$ is smaller or bigger than $f_{1}$, respectively.

Proof. Let $\left\lfloor r_{k} \alpha\right\rfloor=k$, then $0 \leq\left\{r_{k} \alpha\right\} \leq \alpha$ and $\left\{r_{k} \alpha\right\}+\left(a_{1}-1\right) \alpha \leq a_{1} \alpha<1$, hence $t_{k} \geq a_{1}$. Moreover $\left\{r_{k} \alpha\right\}+\left(a_{1}+1\right) \alpha \geq\left(a_{1}+1\right) \alpha>1$, hence $t_{k} \leq a_{1}+1$. Finally $t_{k}=a_{1}+1$ if and only if $\left\{r_{k} \alpha\right\}+a_{1} \alpha<1$, that is if and only if $\left\{r_{k} \alpha\right\}<\left(1-a_{1} \alpha\right)=f_{1}$.

Starting our analysis from $r=0$, we notice that $r_{1}=a_{1}+1$ and $\left\{r_{1} \alpha\right\}=$ $\alpha-f_{1}=f_{0}-f_{1}$. Then $t_{1}=a_{1}+1$ if and only if $f_{0}-f_{1}<f_{1}$, that is if and only if $f_{2}=f_{0}-f_{1}$, that is $a_{2}=1$ (cfr. (2.9) ). If instead $a_{2}>1$ then $\left\{r_{2} \alpha\right\}=\left\{r_{1} \alpha\right\}-f_{1}=f_{0}-2 f_{1}$ and proceeding recursively $\left\{r_{k} \alpha\right\}=$ $f_{0}-k f_{1}>f_{1}$ for all $1 \leq k<a_{2}$, hence $t_{k}=a_{1}$ for all $1 \leq k<a_{2}$. On the other hand $\left\{r_{a_{2}} \alpha\right\}=f_{0}-a_{2} f_{1}=f_{2}<f_{1}$, whence $t_{a_{2}}=a_{1}+1$ and $r_{a_{2}}=q_{2}$, the denominator of the second "fast" rational approximant of $\alpha$.

Let us denote by $\left(r_{k_{j}}\right)$ the sub-sequence of $\left(r_{k}\right)$ such that $t_{k_{j}}=a_{1}+1$ for all $j \geq 0$. So far we have showed that $k_{0}=0, r_{k_{0}}=0$ and $k_{1}=a_{2}, r_{k_{1}}=q_{2}$. We now investigate the following terms of $\left(r_{k_{j}}\right)$. Let $\left(g_{j}\right)$ denote the sequence of "gaps" between subsequent elements of $\left(r_{k_{j}}\right)$ :

$$
g_{j}:=r_{k_{j}}-r_{k_{j-1}}, \quad j \geq 1 .
$$

Given the irrational number $\alpha=\left[a_{1}, a_{2}, \ldots\right]$ and $m \geq 1$, we denote by

$$
\alpha_{m}:=\left[a_{m+1}, a_{m+2}, \ldots\right]=G^{m}(\alpha)
$$


the $m$-th iterate of $\alpha$ under the Gauss map $G:[0,1] \rightarrow[0,1]$ defined by $G(x)=\{1 / x\}$ for $x \neq 0$ and $G(0)=0$. We denote by $p_{n}^{(m)}, q_{n}^{(m)}$ and $f_{n}^{(m)}$ the quantities corresponding to (2.3) and (2.4) for $\alpha_{m}$. It holds

$$
f_{n}^{(m)}=\prod_{k=0}^{n} \alpha_{k+m}
$$

and therefore

$$
\alpha_{r+m}=\frac{f_{r}^{(m)}}{f_{r-1}^{(m)}}, \quad r \geq 0
$$

Let moreover $T_{\alpha}^{(m)}: X \rightarrow X$ denote the rotation with angle $\alpha_{m}$ (so that $T_{\alpha}^{(0)}=T_{\alpha}$ ) and let $t_{k}^{(m)}, r_{k}^{(m)}, r_{k_{j}}^{(m)}$ and $g_{j}^{(m)}$ be the corresponding quantities.

Proposition 2.2. The following relations hold for all $m \geq 0$ :

(i) for all $k \geq 0$

$$
t_{k}^{(m)}= \begin{cases}a_{m+1}+1, & \text { if }\left\{r_{k}^{(m)} \alpha_{m}\right\}<f_{1}^{(m)}=1-a_{m+1} \alpha_{m} \\ a_{m+1}, & \text { otherwise }\end{cases}
$$

(ii) for all $j \geq 1$

$$
g_{j}^{(m)}=r_{k_{j}}^{(m)}-r_{k_{j-1}}^{(m)}= \begin{cases}q_{2}^{(m)}, & \text { if }\left\{(j-1) \alpha_{m+2}\right\}<\left(1-\alpha_{m+2}\right) \\ q_{2}^{(m)}+q_{1}^{(m)}, & \text { otherwise }\end{cases}
$$

(iii) let $\left(j_{h}^{(m)}\right)$ be the subsequence such that $g_{j_{h}}^{(m)}=q_{2}^{(m)}+q_{1}^{(m)}$ for all $h \geq 0$.

$$
\text { Then } j_{0}^{(m)}=a_{m+3}+1 \text { and } j_{h}^{(m)}-j_{h-1}^{(m)}=t_{h}^{(m+2)} \text { for all } h \geq 1 \text {. }
$$

Proof. For notational simplicity' sake we show the results for $m=0$. The general situation is obviously the same.

Point $(i)$ has been proved above.

To prove point (ii) we apply the Three Gap Theorem (see for example [PF]) to the interval $\left(0, f_{1}\right)$. According to this theorem the possible values of the gaps $g_{j}$ between two successive visits of the interval $\left(0, f_{1}\right)$ by the orbit $(j \alpha)$ are given by

$$
g_{j}=r_{k_{j}}-r_{k_{j-1}}= \begin{cases}q_{2}+q_{1}, & \text { with frequency } \frac{f_{2}}{f_{1}} \\ q_{2}, & \text { with frequency } 1-\frac{f_{2}}{f_{1}} .\end{cases}
$$


Let now $\left\{r_{k_{j-1}} \alpha\right\}$ be in $\left(0, f_{1}\right)$. We can repeat the same argument as for $r_{a_{2}}$ to prove that $g_{j}=q_{2}$ if and only if $\left\{r_{k_{j-1}} \alpha\right\}<f_{1}-f_{2}$. Indeed we have $\left\{r_{k_{j-1}+1} \alpha\right\}=\left\{r_{k_{j-1}} \alpha\right\}+f_{0}-f_{1}$, and more generally we can write

$$
\left\{r_{k_{j-1}+h} \alpha\right\}=\left\{r_{k_{j-1}} \alpha\right\}+f_{0}-h f_{1}
$$

for all $h=1,2, \ldots$ such that the r.h.s. remains non-negative. This certainly happens until $h$ reaches the value $a_{2}$, as one readily checks, but for $h=a_{2}+2$ we have $\left\{r_{k_{j-1}} \alpha\right\}+f_{0}-\left(a_{2}+2\right) f_{1}=\left\{r_{k_{j-1}} \alpha\right\}+f_{2}-2 f_{1}<f_{2}-f_{1}<0$ since $\left\{r_{k_{j-1}} \alpha\right\}<f_{1}$. This shows that $a_{2} \leq k_{j}-k_{j-1} \leq a_{2}+1$ and it is a constructive proof of what are the possible values of the gaps $g_{j}$. Now $k_{j}-k_{j-1}$ is equal either to $a_{2}$ or to $a_{2}+1$ (and the gap $g_{j}$ is equal to $q_{2}$ or to $q_{2}+q_{1}$, respectively) if and only if $\left\{r_{k_{j-1}} \alpha\right\}+f_{0}-a_{2} f_{1}$ is smaller or greater than $f_{1}$, respectively. Hence $g_{j}=q_{2}$ if and only if $\left\{r_{k_{j-1}} \alpha\right\}<f_{1}-f_{2}$.

Let us now denote by $\tilde{T}$ the map that acts on $\tilde{X}:=\left[0, f_{1}\right] /\left(0=f_{1}\right)$ as $\tilde{T}:\left\{r_{k_{j}} \alpha\right\} \mapsto\left\{r_{k_{j+1}} \alpha\right\}$, that is the first return map on the interval $\left[0, f_{1}\right]$ for the rotation $T_{\alpha}$. $\tilde{T}$ is isomorphic to the rotation $T_{\alpha}^{(2)}$ of $X$ through the angle $\alpha_{2}=\frac{f_{2}}{f_{1}}=\left[a_{3}, a_{4}, \ldots\right]$. Starting from $r_{k_{0}}=0$, we need to follow the orbit of 0 using the rotation $T_{\alpha}^{(2)}$ and determine the gaps $\left(g_{j}\right)$. We have showed that $g_{j}=q_{2}$ if and only if $\left(T_{\alpha}^{(2)}\right)^{j-1}(0)<1-\frac{f_{2}}{f_{1}}$ or, which is the same by (2.15), $\left\{(j-1) \alpha_{2}\right\}<\left(1-\alpha_{2}\right)$. This proves $(i i)$.

Point (iii) follows by repeating a similar argument for $T_{\alpha}^{(2)}$ on the interval $\left(1-\alpha_{2}, 1\right)$. Again the Three Gap Theorem yields the gaps between two successive visits of the interval $\left(1-\alpha_{2}, 1\right)$ by the orbit $\left((j-1) \alpha_{2}\right)$. Let $\left(j_{h}\right)$ be the subsequence such that $\left\{\left(j_{h}-1\right) \alpha_{2}\right\}>\left(1-\alpha_{2}\right)$, then for each $h \geq 1, j_{h}-j_{h-1}$ is equal either to $a_{3}+1$ or to $a_{3}$, with $j_{0}=a_{3}+1$. Indeed, $\left(j_{0}-1\right) \alpha_{2}=a_{3} \alpha_{2}<1$ but $\left(a_{3}+1\right) \alpha_{2}>1$, therefore $a_{3} \alpha_{2}>\left(1-\alpha_{2}\right)$. This implies that $g_{1}=g_{2}=\cdots=g_{a_{3}}=q_{2}$ and $g_{a_{3}+1}=q_{2}+q_{1}$. Let us remark that $\left\lfloor\left(j_{h-1}-1\right) \alpha_{2}\right\rfloor=h-1$, then $r_{h}^{(2)}=j_{h-1}$, where we recall that $r_{h}^{(2)}$ is defined as the smallest integer such that $\left\lfloor r_{h}^{(2)} \alpha_{2}\right\rfloor=h$. Now $\left\{r_{h}^{(2)} \alpha_{2}\right\}+\left(a_{3}+1\right) \alpha_{2}>1$ hence $\left(j_{h}-1\right)-\left(j_{h-1}-1\right) \leq a_{3}+1$, and $\left\{r_{h}^{(2)} \alpha_{2}\right\}+$ $\left(a_{3}-2\right) \alpha_{2}<\left(a_{3}-1\right) \alpha_{2}<1-\alpha_{2}$ hence $\left(j_{h}-1\right)-\left(j_{h-1}-1\right) \geq a_{3}$. Moreover $\left\{r_{h}^{(2)} \alpha_{2}\right\}<f_{1}^{(2)}=1-a_{3} \alpha_{2}$ if and only if $\left\{r_{h}^{(2)} \alpha_{2}\right\}+\left(a_{3}-1\right) \alpha_{2}<1-\alpha_{2}$, hence if and only if $\left(j_{h}-1\right)-\left(j_{h-1}-1\right)=a_{3}+1$. This shows that for all $h \geq 1 j_{h}-j_{h-1}$ is equal to $t_{h}^{(2)}$ and both are equal either to $a_{3}+1$ or to $a_{3}$.

The proof given above brings out the renormalization argument mentioned in the Introduction and which will be fully developed in the next section. 
According to the above discussion, since $j_{0}=a_{3}+1$, the values of the sequence $r_{k_{j}}$, with $1 \leq j \leq a_{3}+1$, are given by

$$
0, q_{2}, 2 q_{2}, \ldots, a_{3} q_{2}, a_{3} q_{2}+q_{2}+q_{1}=q_{3}+q_{2} .
$$

Note that $k_{a_{3}+1}=p_{3}+p_{2}$. To continue the determination of the numbers $r_{k_{j}}$ we have to use the knowledge of the following $j_{h}$ and by point (iii) this is equivalent to repeat the argument above for the rotation $T_{\alpha}^{(2)}$.

We need the Ostrowski representation of an integer number [PF]: given an irrational number $\alpha \in(0,1)$ with partial quotients $\left(a_{h}\right)$ and denominators $\left(q_{h}\right)$ of its rational approximants, any positive integer $r$ can be written in a unique way in the form

$$
r=\sum_{h \geq 0}^{N} c_{h} q_{h} \quad \text { with } \quad 0 \leq c_{h} \leq a_{h+1} \quad \text { and } \quad c_{h-1}=0 \quad \text { if } \quad c_{h}=a_{h+1}
$$

for some integer $N$. We call $N$ the order of the integer $r$, denoted as $N=$ $\operatorname{ord}(r)$.

Theorem 2.3. Given a positive integer $r$, we have $r=r_{k_{j}}$ for some $j>0$ if and only if in the Ostrowski representation of $r$ we have: $c_{0}=c_{1}=0$ and $\min \left\{h: c_{h}>0\right\} \geq 2$ and even. Moreover, a positive integer $r$ is of the form $r_{k}$, for some $k$, if and only if either $r=r_{k_{j}}$ for some $j>0$ or $r=r_{k_{j}}+c_{1} q_{1}+1$ for some $j>0$ and $1 \leq c_{1} \leq a_{2}$.

Proof. We have verified the first part of the thesis for $r \leq q_{3}+q_{2}$, finding $r_{k_{j}}$ with $j=1, \ldots, a_{3}+1$. To continue, by Proposition 2.2(iii) we have to study the sequence $t_{h}^{(2)}$, that is the sequence $t_{h}$ for the angle $\alpha_{2}=\left[a_{3}, a_{4}, \ldots\right]$. The first $\left(a_{4}+1\right)$ values are

$$
a_{3}+1, a_{3}, a_{3}, \ldots, a_{3}, a_{3}+1
$$

as obtained by part (i) and (ii) of Proposition 2.2 for $m=2$. This leads to the computation of $r_{k_{j}}$ up to $\left(q_{4}+q_{3}+q_{2}\right)$. What happens after depends on whether $r_{k_{2}}^{(2)}$ is $q_{2}^{(2)}=\left(a_{4} a_{3}+1\right)$ or $q_{2}^{(2)}+q_{1}^{(2)}=\left(a_{4} a_{3}+a_{3}+1\right)$. We already solved this problem for $r_{k_{j}}^{(0)}$ up to $j=a_{3}+1$. Hence in the same way we can solve the problem for $r_{k_{j}}^{(2)}$ up to $j=a_{3}^{(2)}+1=a_{5}+1$. This implies the thesis up to $\left(q_{5}+q_{2}\right)$.

The subsequent steps follow by repeating the same argument as before, where for all $i \geq 2$, the denominators $q_{2 i}$ and $q_{2 i+1}$ substitute $q_{4}$ and $q_{5}$. Whence the form of the integers $r_{k_{j}}$ follows by induction on $i \geq 2$. 
To prove the result for $r_{k}$, simply notice that if $r_{k}$ is not $r_{k_{j}}$, then it is obtained from one of the $r_{k_{j}}$ by adding $q_{1}$ as many times as needed, since $t_{k} \geq q_{1}$, hence there are at least $q_{1}$ iterations before $\lfloor r \alpha\rfloor$ increases. Moreover the iterations can't be more than $q_{1}$ because $r_{k}$ is not $r_{k_{j}}$. This finishes the proof.

We finally point out that the following relation between an integer $r_{k_{j}}$ and its index $j$ is in force: first, for $j>0$ we have

$$
r_{k_{j}}=\sum_{h \geq 2} c_{h} q_{h} \quad \Rightarrow \quad k_{j}=\sum_{h \geq 2} c_{h} p_{h}
$$

Second, replacing $p_{3}$ and $p_{2}$ in $k_{j}$ with $a_{3}$ and 1 , respectively, and using the definition of the numbers $q_{h}^{(2)}$, one obtains inductively

$$
j=\sum_{h \geq 2} c_{h} q_{h-2}^{(2)}=\sum_{h \geq 2} c_{h}\left(q_{h}-a_{1} p_{h}\right) .
$$

In the following, besides $\alpha_{m}=G^{m}(\alpha)$ we will also need the numbers

$$
\bar{\alpha}_{m}:=\left[a_{m+1}-1, a_{m+2}, \ldots\right]=\frac{G^{m}(\alpha)}{1-G^{m}(\alpha)} .
$$

We remark that if $a_{m+1}=1$ then $\bar{\alpha}_{m}=\alpha_{m+2}$. Let us denote by $\bar{T}_{\alpha}^{(m)}: X \rightarrow$ $X$ the rotation of angle $\bar{\alpha}_{m}$ and $\bar{p}_{n}^{(m)}, \bar{q}_{n}^{(m)}, \bar{f}_{n}^{(m)}$ the corresponding quantities (cfr. (2.3) and (2.4) ). A simple inductive argument shows that in the case $m=1$, if $r_{k_{j}}$ is defined as above, then

$$
k_{j}-j= \begin{cases}\sum_{h \geq 2} c_{h} \bar{q}_{h-1}^{(1)}, & \text { if } a_{2} \neq 1, \\ \sum_{h \geq 3} c_{h} \bar{q}_{h-3}^{(1)}, & \text { if } a_{2}=1 .\end{cases}
$$

Whereas for the sequence $\left(\bar{f}_{n}^{(m)}\right)$ it holds for all $m \geq 1$ and all $n \geq 0$

$$
\bar{f}_{n}^{(m)}= \begin{cases}\frac{f_{n+1}^{(m-1)}}{f_{0}^{(m-1)}-f_{1}^{(m-1)}}, & \text { if } a_{2} \neq 1, \\ \frac{f_{n+3}^{(m-1)}}{f_{2}^{(m-1)}}, & \text { if } a_{2}=1 .\end{cases}
$$

We end this section by giving the following version of a standard expansion of a real number $\beta \in(0,1)$ in terms of the numbers $f_{n}$ defined in (2.4) for a fixed irrational number $\alpha$ with partial quotients $\left(a_{k}\right)$ (see, e.g., [PF], Sect. $6.4)$ 
Proposition 2.4. For all $\beta \in(0,1)$ there exists a unique sequence of integers $\left(b_{k}\right)$ such that: (i) $\beta=\sum_{k=0}^{\infty} b_{k} f_{k}$; (ii) $0 \leq b_{k} \leq a_{k+1}$ for all $k \geq 0$; (iii) $b_{k}=a_{k+1}$ implies $b_{k+1}=0$. Moreover the coefficients $\left(b_{k}\right)$ are definitively null if and only if $\beta \in \mathbb{Z}+\alpha \mathbb{Z}$.

Proof. By definition, $\left(f_{k}\right)$ is a monotonically decreasing sequence of positive real numbers. The sequence $\left(b_{k}\right)$ is constructed by a greedy algorithm: let

$$
b_{0}:=\left\lfloor\frac{\beta}{\alpha}\right\rfloor, \quad \beta_{1}:=\beta-b_{0} \alpha,
$$

where we recall $\alpha=f_{0}$. Note that $\beta<1$ implies $b_{0} \leq a_{1}$ and $\beta_{1}<f_{0}$. Then we can define by induction for all $k \geq 1$

$$
b_{k}:=\left\lfloor\frac{\beta_{k}}{f_{k}}\right\rfloor, \quad \quad \beta_{k+1}:=\beta_{k}-b_{k} f_{k}=\beta-\sum_{i=0}^{k} b_{i} f_{i} .
$$

By definition of $b_{k}$, it holds $\beta_{k}<f_{k-1}$, hence $\beta=\lim _{k} \sum_{i=0}^{k} b_{i} f_{i}$ and $b_{k} \leq$ $a_{k+1}$ (see equation (2.9)). Moreover, $b_{k}=a_{k+1}$ implies $\beta_{k+1}<f_{k-1}-$ $a_{k+1} f_{k}=f_{k+1}$ (see equation (2.8) $)$, hence $b_{k+1}=0$. This proves part (i), (ii) and (iii).

Let now $b_{k}=0$ for all $k>\bar{k}$, for some integer $\bar{k}$. Then $\beta=\sum_{i=0}^{\bar{k}} b_{i} f_{i}$ and $f_{k} \in \mathbb{Z}+\alpha \mathbb{Z}$ for all $k \geq 0$ imply that $\beta \in \mathbb{Z}+\alpha \mathbb{Z}$. Conversely, let $\beta=t+\alpha s$ for $t, s \in \mathbb{Z}$. Since $\beta \in(0,1)$, if $t=0$ then $0 \leq s \leq a_{1}$, hence $b_{0}=s$ and $\beta_{1}=0$. This implies $b_{k}=0$ for all $k \geq 1$. Let now $t>0$ so that $s<0$. If we let $m=\max \{r \in \mathbb{N}:\lfloor r \alpha\rfloor=t-1\}$, then we can write $\beta=t-m \alpha+(m-|s|) \alpha$, where $m-|s| \leq a_{1}$. Using the expansion of equation (3.2), we can write $m=r_{k_{j}}+R_{1} q_{1}$ for some $r_{k_{j}}=\sum_{h=2}^{N} c_{h} q_{h}$ and $0 \leq R_{1} \leq a_{2}+1$. Notice that $R_{0}=0$ by the definition of $m$. From this, using equation (2.17), we obtain $t=k_{j}+R_{1}$ and therefore

$$
\beta=\sum_{h=2}^{N}(-1)^{h+1} c_{h} f_{h}+R_{1} f_{1}+(m-|s|) f_{0}
$$

From the definition of $b_{k}$ one immediately sees that $b_{k}=0$ for all $k>N$. The same argument works for the case $t<0$.

\section{The growth of $S_{n}(\alpha)$}

We now use the sequence $\left(t_{k}\right)$ to study the behaviour of $S_{n}(\alpha)$ and whence the diffusive properties of the corresponding walk. 
We have showed that $t_{k}$ is equal either to $a_{1}$ or to $a_{1}+1$. Therefore, according to whether $a_{1}$ is even or odd, only the iterations for which $t_{k}=a_{1}+1$ or $t_{k}=a_{1}$, respectively, are important for the growth behaviour.

Let us consider first of all the case $a_{1}$ even. In this case, if $t_{k}=a_{1}$ then $\sum_{i=r_{k}}^{r_{k}+a_{1}-1} s(i \alpha)=0$, hence we can neglect these terms, since the "walker" associated to $S_{n}(\alpha)$ would simply take $a_{1}$ steps to start from $S_{r_{k}-1}(\alpha)$ and come back to the same point, after having reached the point $S_{r_{k}-1}(\alpha)+\frac{a_{1}}{2}$. Hence we can restrict ourselves to the study of the sequence $\{r \alpha\}$ with $r_{k_{j}} \leq r \leq r_{k_{j}}+a_{1}$, where we recall that the sub-sequence $\left(r_{k_{j}}\right)$ corresponds to $t_{k_{j}}=a_{1}+1$. In these cases $\sum_{i=r_{k_{j}}}^{r_{k_{j}}+a_{1}} s(i \alpha)= \pm 1$, according to whether the number $\left\{r_{k_{j}} \alpha+\frac{a_{1}}{2} \alpha\right\}$ is $<\frac{1}{2}$ or $>\frac{1}{2}$, respectively, that is whether $\left\{r_{k_{j}} \alpha\right\}<$ $\frac{1}{2} f_{1}$ or $>\frac{1}{2} f_{1}$. In view of the analysis made in the previous section, given the first return map $\tilde{T}$ on the interval $\left(0, f_{1}\right)$, and its isomorphism with the rotation $T_{\alpha}^{(2)}$ on $X$, we conclude that

$$
\sum_{i=r_{k_{j}}}^{r_{k_{j}}+a_{1}} s\left(T_{\alpha}^{i}(0)\right)=1 \Longleftrightarrow\left(T_{\alpha}^{(2)}\right)^{j}(0)<\frac{1}{2} .
$$

Using this fact we now study the relation between the sequences $S_{n}(\alpha)$ and $S_{n}\left(\alpha_{2}\right)$. We obtain that for all $r \geq 0$ it holds

$$
a_{1} \text { even } \Longrightarrow S_{r}(\alpha)=S_{j(r)}\left(\alpha_{2}\right)+\tilde{S}(r)
$$

where $j(r)$ and $\tilde{S}(r)$ are computed in the following way. Let us write $r$ in the form

$$
r=r_{k_{j}}+R_{1} q_{1}+R_{0}
$$

with $R_{1} q_{1}+R_{0}<r_{k_{j+1}}-r_{k_{j}}, 0 \leq R_{1} \leq a_{2}+1$ and $0 \leq R_{0}<q_{1}$. We remark that this can be different from the Ostrowski representation of $r$, since it can be $R_{1}=a_{2}+1$. However the order of $r$ is equal to that of $r_{k_{j}}$ for $j>0$. We have

$$
j(r)=\max \left\{\bar{j} \geq 0: r_{k_{\bar{j}}}<r-R_{0}\right\}=\max \left\{j+\operatorname{sgn}\left(R_{1}\right)-1,0\right\}
$$

and

$$
\tilde{S}(r)= \begin{cases}\sum_{i=r-R_{0}+1}^{r} s(\{i \alpha\}) & \text { if } R_{0}>0, R_{1}>0, \\ \sum_{i=r-R_{0}}^{r} s(\{i \alpha\}) & \text { if } R_{0}>0, R_{1}=0, \\ 0 & \text { if } R_{0}=0, R_{1}>0, \\ s(\{r \alpha\}) & \text { if } R_{0}=0, R_{1}=0 .\end{cases}
$$


We remark that using equations (2.17) and (2.18) it is possible to obtain $j$ from the knowledge of $r_{k_{j}}$. Moreover $0 \leq \tilde{S}(r) \leq 1+\frac{a_{1}}{2}$ for all $r \geq 0$, hence the growth behaviour of $S_{n}(\alpha)$ only depends on that of $S_{n}\left(\alpha_{2}\right)$.

The case $a_{1}$ odd is in some sense complementary to the previous one. Indeed, in this case, we obviously have $\sum_{i=k_{k_{j}}}^{r_{k_{j}}+a_{1}} s\left(T_{\alpha}^{i}(0)\right)=0$, whereas for $k$ such that $t_{k}=a_{1}$ we have $\sum_{i=r_{k}}^{r_{k}+a_{1}-1} s\left(T_{\alpha}^{i}(0)\right)= \pm 1$ according to whether $\left\{r_{k} \alpha+\right.$ $\left.\frac{a_{1}-1}{2} \alpha\right\}<\frac{1}{2}$ or $>\frac{1}{2}$. We would like to construct an induced map on some interval of $X$, to connect the values of $\sum_{i=r_{k}}^{r_{k}+a_{1}-1} s\left(T_{\alpha}^{i}(0)\right)$ to a suitable orbit of such induced map. To this aim we notice that the point $\left\{r_{k} \alpha+\frac{a_{1}-1}{2} \alpha\right\}$ belongs to the interval $J:=\left(\frac{1}{2}-\frac{1}{2}\left(f_{0}-f_{1}\right), \frac{1}{2}+\frac{1}{2}\left(f_{0}-f_{1}\right)\right)$ for all $k \geq 0$ such that $t_{k}=a_{1}$. This follows immediately from the following remarks:

1. $\left\{r_{k} \alpha\right\}>f_{1}$ and $f_{1}+\frac{a_{1}-1}{2} \alpha=\frac{1}{2}-\frac{1}{2}\left(f_{0}-f_{1}\right)$;

2. $\left\{r_{k} \alpha+\frac{a_{1}-1}{2} \alpha\right\}<f_{1}+\frac{a_{1}+1}{2} \alpha-f_{1}$, since $f_{1}+\frac{a_{1}+1}{2} \alpha>\left\{r_{k_{j}} \alpha+\frac{a_{1}-1}{2} \alpha\right\}$ for all $k_{j}$, and $\frac{a_{1}+1}{2} \alpha=\frac{1}{2}+\frac{1}{2}\left(f_{0}-f_{1}\right)$.

Moreover the two estimates in 1. and 2. are sharp.

From the definition of the interval $J$, it also follows that $\left\{r_{k_{j}} \alpha+r \alpha\right\} \notin J$ for all $r=1, \ldots, a_{1}$, since $\left\{r_{k_{j}} \alpha\right\} \in\left(0, f_{1}\right)$ implies that $\left\{r_{k_{j}} \alpha+\frac{a_{1}-1}{2} \alpha\right\}<$ $\frac{1}{2}-\frac{1}{2}\left(f_{0}-f_{1}\right)$ and $\left\{r_{k_{j}} \alpha+\frac{a_{1}+1}{2} \alpha\right\}>\frac{1}{2}+\frac{1}{2}\left(f_{0}-f_{1}\right)$. Hence we can consider the first return map $\bar{T}$ of $T_{\alpha}$ to the interval $J$, and obtain that $\bar{T}$ is isomorphic to the inverse of the rotation $\bar{T}_{\alpha}^{(1)}$ on $X$, that is the rotation of angle

$$
-\bar{\alpha}_{1}=-\frac{f_{1}}{f_{0}-f_{1}} \text {. }
$$

Let now $\left(k_{i}\right)$ be the sub-sequence such that $t_{k_{i}}=a_{1}$ for all $i \geq 1$, then

$$
\sum_{r=r_{k_{i}}}^{r_{k_{i}}+a_{1}-1} s\left(T_{\alpha}^{r}(0)\right)=1 \Longleftrightarrow\left(\bar{T}_{\alpha}^{(1)}\right)^{i}(0)<\frac{1}{2}
$$

We now want to give an analogous equation of (3.1). In this case we have to neglect $\left(\bar{T}_{\alpha}^{(1)}\right)^{0}(0)=0$, since we start with $i=1$, hence we obtain that for all $r \geq 0$

$$
a_{1} \text { odd } \Longrightarrow S_{r}(\alpha)=S_{i(r)}\left(-\bar{\alpha}_{1}\right)-1+\tilde{S}(r)
$$

where $\tilde{S}(r)$ is the same as in equation (3.1) and $i(r)$ is computed in the following way. Let us write again $r$ as in equation (3.2), then

$$
i(r)=k_{j}-j+\max \left\{\left(R_{1}-1\right), 0\right\}
$$


where we recall equations (2.17), (2.18) and (2.20).

Again $\tilde{S}(r)$ is uniformly bounded so that the diffusive properties of $S_{n}(\alpha)$ depend only on those of $S_{n}(\bar{\alpha})$. Moreover we note that for all $n \geq 0$ we have

$$
S_{n}\left(-\bar{\alpha}_{1}\right)-1=-\left(S_{n}\left(\bar{\alpha}_{1}\right)-1\right)
$$

In conclusion, we have showed that, as far as the diffusive properties are concerned, the walk $\left(S_{n}(\alpha)\right)$ is equivalent to a "renormalized" walk $\left(S_{R(n)}(\beta)\right)$, where the values of $R(n)$ and $\beta$ depend on the parity of $a_{1}$, the first partial quotient of the number $\alpha$.

Equations (3.1) and (3.5) lead by iteration to an explicit expression for $S_{n}(\alpha)$ only in terms of the $\left(a_{k}\right)$. A tentative result in this direction was given in [So]. About growth estimates, let us see how this argument leads to precise estimates on the behaviour of maxima and minima of $S_{n}(\alpha)$.

Proposition 3.1. Given $\alpha=\left[a_{1}, a_{2}, \ldots\right] \in(0,1)$, let $r=r_{k_{j}}+R_{1} q_{1}+R_{0}$ for some $j \geq 0$ as in equation (3.2). If $a_{1}$ is even then

$$
\begin{gathered}
0 \leq \max _{0 \leq n \leq r} S_{n}(\alpha)-\left(\max _{0 \leq m \leq j(r)} S_{m}\left(\alpha_{2}\right)+\frac{a_{1}}{2}\right) \leq 1 \\
\min _{0 \leq n \leq r} S_{n}(\alpha)=\min _{0 \leq m \leq j(r)} S_{m}\left(\alpha_{2}\right)
\end{gathered}
$$

where $\alpha_{2}=\left[a_{3}, a_{4}, \ldots\right]$ and $j(r)$ is given in (3.3). If instead $a_{1}$ is odd then

$$
\begin{gathered}
0 \leq \max _{0 \leq n \leq r} S_{n}(\alpha)-\left(1-\min _{0 \leq m \leq i(r)} S_{m}\left(\bar{\alpha}_{1}\right)+\frac{a_{1}-1}{2}\right) \leq 1 \\
\min _{0 \leq n \leq r} S_{n}(\alpha)=1-\max _{0 \leq m \leq i(r)} S_{m}\left(\bar{\alpha}_{1}\right)
\end{gathered}
$$

where $\bar{\alpha}_{1}=\left[a_{2}-1, a_{3}, \ldots\right]$ and $i(r)$ is given in (3.5).

Moreover in the case $a_{1}$ even, the difference between maxima is equal to 1 only if $R_{1}=0$ and $R_{0} \geq \frac{a_{1}}{2}$.

Proof. Let us consider first the case $a_{1}$ even. The result is a direct consequence of equation (3.1) and the relation $0 \leq \tilde{S}(r) \leq 1+\frac{a_{1}}{2}$.

For the case $a_{1}$ odd, the proof follows from equations (3.5) and (3.7).

We point out that, since $j(r)$ and $i(r)$ are explicitly computable from $r$, one can iterate the renormalization argument in such a way that at the end of the process the maxima and minima of the walk $S_{n}(\alpha)$ will be explicitly 
computable linear combinations of the partial quotients of $\alpha$. To this end we observe that in order to apply the argument to $S_{m}\left(\alpha_{2}\right)$ it is enough to notice that in equation (2.18) the number $j$ is obtained as a linear combination of $a_{3}$ and 1 , which are nothing but $q_{1}^{(2)}$ and $q_{0}^{(2)}$ respectively (we are using the notations of Proposition 2.2). Therefore $j$ can be expressed with respect to $\alpha_{2}$ in the form $r_{k_{l}}^{(2)}+R_{1}^{(2)} q_{1}^{(2)}+R_{0}^{(2)}$, and the iteration can proceed. We thus obtain the following,

Theorem 3.2. Let the partial quotients $\left(a_{2 i+1}\right)$ be even for all $i \geq 0$. If $r=r_{k_{j}}+R_{1} q_{1}+R_{0}$ for some $j \geq 0$ and $\operatorname{ord}(r)=N$, then

$$
\begin{gathered}
\frac{1}{2} \sum_{i=0}^{\frac{N-2}{2}} a_{2 i+1} \leq \max _{0 \leq n \leq r} S_{n}(\alpha) \leq \frac{N}{2}+\frac{1}{2} \sum_{i=0}^{\frac{N-2}{2}} a_{2 i+1} \\
\min _{0 \leq n \leq r} S_{n}(\alpha)=1
\end{gathered}
$$

This theorem implies that the diffusion properties of $S_{n}(\alpha)$ depend only weakly on the partial quotients $\left(a_{2 i}\right)$. In particular, for all $\alpha$ with fixed partial quotients $\left(a_{2 i+1}\right)$, even for all $i \geq 0$, the sequence $S_{n}(\alpha)$ grows with the same rate, and what changes is the number of fluctuations.

The situation is more cumbersome for numbers $\alpha$ with odd partial quotients in an odd position. This would imply to change the kind of "renormalization", and also partial quotients with even position become important. However, we can make some computations for particular cases.

EXAmPle. Let $\alpha=[a, a, a, \ldots]$ with $a$ odd. Then the first renormalization leads to $\bar{\alpha}_{1}=[a-1, a, a, \ldots]$. This fact implies that two different situations occur for $a=1$ and $a>1$. Hence the sequence $S_{n}(\alpha)$ with $\alpha$ the golden ratio $\frac{\sqrt{5}-1}{2}$ has peculiar properties.

Let us first consider the case $a>1$. From Proposition 3.1 it follows that

$$
\begin{gathered}
\max _{0 \leq n \leq r} S_{n}(\alpha) \leq 2+\frac{a-1}{2}-\min _{0 \leq m \leq j(i(r))} S_{m}(\alpha) \leq \\
\leq 2+(a-1)+\max _{0 \leq n \leq j(i(j(i(r))))} S_{n}(\alpha)
\end{gathered}
$$

where $\operatorname{ord}(j(i(j(i(r)))))=\operatorname{ord}(r)-6$. Therefore, if $\operatorname{ord}(r)=6 k$, repeating the same argument from below we have

$$
\frac{(a-1)}{6} \operatorname{ord}(r) \leq \max _{0 \leq n \leq r} S_{n}(\alpha) \leq \frac{(a+1)}{6} \operatorname{ord}(r)
$$


For example, if $a=3$ then $\alpha=\frac{\sqrt{13}-3}{2}$, and

$$
\frac{1}{3 \log \left(\frac{\sqrt{13}+3}{2}\right)} \leq \limsup _{r \rightarrow \infty} \frac{\max _{0 \leq n \leq r} S_{n}\left(\frac{\sqrt{13}-3}{2}\right)}{\log r} \leq \frac{2}{3 \log \left(\frac{\sqrt{13}+3}{2}\right)}
$$

Let us consider now $a=1$. From Proposition 3.1 it follows that

$$
\max _{0 \leq n \leq r} S_{n}\left(\frac{\sqrt{5}-1}{2}\right) \leq 1+\max _{0 \leq m \leq i(i(r))} S_{m}\left(\frac{\sqrt{5}-1}{2}\right)
$$

and $\operatorname{ord}(i(i(r)))=\operatorname{ord}(r)-6$. Hence

$$
\limsup _{r \rightarrow \infty} \frac{\max _{0 \leq n \leq r} S_{n}\left(\frac{\sqrt{5}-1}{2}\right)}{\log r} \leq \frac{1}{6 \log \left(\frac{\sqrt{5}+1}{2}\right)} .
$$

\section{Generalisations to other orbits and applications}

We now use the formalism developed in the previous sections to analyse the behaviour of the following quantity

$$
d_{n}(\alpha, \beta):=\sum_{r=0}^{n-1} \chi_{[0, \beta)}(\{r \alpha\})-\beta n
$$

for points $\beta \in(0,1)$. We call $d_{n}(\alpha, \beta)$ the relative discrepancy of $\alpha$ with respect to $\beta$. The term is justified by the usual definition of discrepancy of the sequence $(n \alpha)$ as

$$
D_{n}^{*}(\alpha):=\sup _{\beta \in(0,1)}\left|\frac{1}{n} d_{n}(\alpha, \beta)\right|
$$

We first give an iterative argument to compute the relative discrepancies $d_{n}(\alpha, \beta)$. This is useful to give an explicit expression for the diffusion of the orbit of a general point $\beta \in(0,1)$ for a rotation $T_{\alpha}$, that is

$$
S_{n}(\alpha, \beta):=\sum_{r=0}^{n} s(\{r \alpha+\beta\})
$$

(see (1.1) $)$. 
Given $\alpha=\left[a_{1}, a_{2}, \ldots\right]$, we recall the notations

$$
\begin{gathered}
\alpha_{m}=G^{m}(\alpha)=\left[a_{m+1}, a_{m+2}, \ldots\right] \\
\bar{\alpha}_{m}=\frac{G^{m}(\alpha)}{1-G^{m}(\alpha)}=\left[a_{m+1}-1, a_{m+2}, \ldots\right]
\end{gathered}
$$

where $G$ is the Gauss map, as well as the sequences $p_{h}, q_{h}, f_{h}$ and $r_{k_{j}}$ associated to $\alpha$. Let us fix $\beta \in(0,1)$ and recall its expansion $\beta=\sum_{k} b_{k} f_{k}$ as well as the numbers $\beta_{m}$ given in (2.22). Let us define for all $m \geq 1$

$$
\beta^{m}:=\frac{\beta_{m}}{f_{m}} \quad \bar{\beta}^{m}:=\frac{\beta_{m}-f_{m}}{f_{m-1}-f_{m}}
$$

Proposition 4.1. For a given $n \in \mathbb{N}$, let us write $n-1=r_{k_{j}}+R_{1} q_{1}+R_{0}$ as in equation (3.2) with ord $(n)=N$. Let $j(n-1)$ and $i(n-1)$ be defined as in equations (3.3) and (3.6) respectively, and let $\tilde{S}(n-1)$ be defined as in equation (3.4). If we define

$$
S(n, \alpha, \beta)=\tilde{S}(n-1)-\beta\left(R_{0}+1-\operatorname{sgn}\left(R_{1}\right)\right)
$$

and write $r_{k_{j}}=\sum_{h=2}^{N} c_{h} q_{h}$, then

$$
d_{n}(\alpha, \beta)=S(n, \alpha, \beta)+\left\{\begin{array}{l}
-\frac{\left(b_{0}-a_{1} \beta\right)}{f_{1}} C(\alpha, n)+d_{j(n-1)+1}\left(\alpha_{2}, \beta^{1}\right) \\
\frac{\left(b_{0}-a_{1} \beta+1-\beta\right)}{f_{0}-f_{1}} C(\alpha, n)+\bar{\beta}^{1}+d_{i(n-1)+1}^{c}\left(\bar{\alpha}_{1}, \bar{\beta}^{1}\right)
\end{array}\right.
$$

where the first formulation is valid if $b_{1}=0$ and the second otherwise, the constant $C(\alpha, n)$ does not depend on $\beta$ and is given by

$$
C(\alpha, n):=\alpha \operatorname{sgn}\left(R_{1}\right)-R_{1} f_{1}+\sum_{h=2}^{N}(-1)^{h} c_{h} f_{h}
$$

and $d^{c}(\cdot)$ means that in the definition of $d(\cdot)$ we use the indicator function of the interval $\left(1-\beta^{1}, 1\right]$.

Proof. The main idea is to use the partition of the sequence $\{r \alpha\}$ using the sequence $\left\{r_{k}\right\}$. Indeed, as in the treatment of the diffusion, we have that for all $k \geq 0$

$$
\sum_{r=r_{k}}^{r_{k+1}-1} \chi_{[0, \beta)}(\{r \alpha\})= \begin{cases}b_{0}+1 & \text { if }\left\{r_{k} \alpha\right\}<\beta_{1} \\ b_{0} & \text { otherwise }\end{cases}
$$


By lemma 2.1, if $b_{1}=0$, that is $\beta_{1}<f_{1}$, the first case is possible only if $k=k_{j}$ for some $j \geq 0$. In this case we have

$$
\sum_{r=0}^{n-1} \chi_{[0, \beta)}(\{r \alpha\})=\tilde{S}(n-1)+b_{0}\left(k_{j}+R_{1}\right)+\sum_{j=0}^{j(n-1)} \chi_{\left[0, \beta^{1}\right)}\left(\left\{j \alpha_{2}\right\}\right)
$$

where the last term accounts for the relation

$$
\sum_{r=r_{k_{j}}}^{r_{k_{j}+q_{1}}} \chi_{[0, \beta)}(\{r \alpha\})=b_{0}+\chi_{\left[0, \beta_{1}\right)}\left(\left\{r_{k_{j}} \alpha\right\}\right)
$$

and uses the isomorphism between the first return function to the set $\left(0, f_{1}\right)$ and the rotation of angle $\alpha_{2}$ on the unit circle. Moreover let us write

$$
\beta n=\beta\left(R_{0}+1-\operatorname{sgn}\left(R_{1}\right)\right)+\beta\left(j+\operatorname{sgn}\left(R_{1}\right)\right)+\beta\left(r_{k_{j}}+R_{1} q_{1}-j\right)
$$

where, we recall, $j(n-1)=j+\operatorname{sgn}\left(R_{1}\right)-1(\operatorname{cfr}$. (3.3) $)$ and $\left(r_{k_{j}}+R_{1} q_{1}-j\right)=$ $a_{1}\left(k_{j}+R_{1}\right)$ (cfr. (2.17) and (2.18) $)$. The claim now follows by evaluating

$$
k_{j}+R_{1}-\frac{\alpha}{f_{1}}\left(j+\operatorname{sgn}\left(R_{1}\right)\right)=-\frac{C(\alpha, n)}{f_{1}} .
$$

A similar argument can be applied if $b_{1}>0$, using the fact that the first return map to the interval $\left(f_{1}, \alpha\right)$ is isomorphic to the inverse of the rotation on the unit interval with angle $\bar{\alpha}_{1}$. This leads to the term $d^{c}(\cdot)$.

The previous result shows that to evaluate $d_{n}(\alpha, \beta)$ we have to repeat the same argument for $d_{j(n-1)+1}$ and $d_{i(n-1)+1}$, respectively. To this end we point out that the same argument as before yields for general $\alpha$ and $\beta$

$$
d_{n}^{c}(\alpha, \beta)=S^{c}(n, \alpha, \beta)+\left\{\begin{array}{l}
-\frac{\left(b_{0}-a_{1} \beta\right)}{f_{1}} C(\alpha, n)+d_{j(n-1)+1}^{c}\left(\alpha_{2}, \beta^{1}\right) \\
\frac{\left(b_{0}-a_{1} \beta+1-\beta\right)}{f_{0}-f_{1}} C(\alpha, n)+\bar{\beta}^{1}+d_{i(n-1)+1}\left(\bar{\alpha}_{1}, \bar{\beta}^{1}\right)
\end{array}\right.
$$

where $S^{c}(n, \alpha, \beta)$ is obtained by using the indicator function of the interval $(1-\beta, 1]$ in $\tilde{S}(n-1)$.

Going on, we see that we can repeat the same argument until the "renormalized" rotation that we obtain does not have enough iterates to be renormalized again. When this happens is up to the order of $n$ and to $\beta$, which implies the "renormalization path" that have to be followed. Let us see 
how the algorithm to choose the new angle of rotation and the new interval works. We have already seen that the first step is

$$
(\alpha, \beta) \longrightarrow \begin{cases}\left(\alpha_{2}, \beta^{1}\right) & \text { if } b_{1}=0 \\ \left(\bar{\alpha}_{1}, \bar{\beta}^{1}\right)^{c} & \text { if } b_{1}>0\end{cases}
$$

and that two subsequent $(\cdot)^{c}$ cancel out (since they are generated by two inversion in the rotations). By straightforward computation, one can readily verify that the general scheme is as follows: the starting point is always of the form $\left(\alpha_{m}, \beta^{m-1}\right)$ or $\left(\bar{\alpha}_{m}, \bar{\beta}^{m}\right)$, and for all $m \geq 2$ we have

$$
\left(\alpha_{m}, \beta^{m-1}\right) \longrightarrow \begin{cases}\left(\alpha_{m+2}, \beta^{m+1}\right), & \text { if } b_{m+1}=0 \\ \left(\bar{\alpha}_{m+1}, \bar{\beta}^{m+1}\right)^{c}, & \text { if } b_{m+1}>0\end{cases}
$$

the same holding true for $\left(\bar{\alpha}_{m}, \bar{\beta}^{m}\right)$.

To conclude, we remark that the constants $C\left(\alpha_{m}, n\right)$ and $C\left(\bar{\alpha}_{m}, n\right)$ are the same as in Proposition 4.1, with the values $f_{n}^{(m)}$ and $\bar{f}_{n}^{(m)}$ computed using equation (2.14) and (2.21), respectively. Furthermore, the coefficients multiplying $C\left(\alpha_{m}, n\right)$ and $C\left(\bar{\alpha}_{m}, n\right)$ at each step satisfy the following

Lemma 4.2. For all $\alpha$ and $\beta$ it holds:

(i) if $b_{m+1}=0$ then $-a_{1}^{(m)} \leq \frac{\left(b_{0}^{m}-a_{1}^{(m)} \beta^{m-1}\right)}{f_{1}^{(m)}} \leq a_{1}^{(m)}$;

(ii) if $b_{m+1}>0$ then $-a_{1}^{(m)} \leq \frac{\left(b_{0}^{m}-a_{1}^{(m)} \beta^{m-1}+1-\beta^{m-1}\right)}{f_{0}^{(m)}-f_{1}^{(m)}} \leq a_{1}^{(m)}$;

(iii) for all $m \geq 0$ and all $n$, it holds $0<C\left(\alpha_{m}, n\right)<\alpha_{m}$.

The same relations hold if we consider the corresponding quantities for $\bar{\alpha}_{m}$ and $\bar{\beta}^{m}$.

Using the above algorithm we are able to obtain the actual values of $d_{n}(\alpha, \beta)$. To obtain growth estimates we write

$$
d_{n}(\alpha, \beta)=\mathcal{C}(n, \alpha, \beta)+\mathcal{S}(n, \alpha, \beta)+\mathcal{B}(n, \alpha, \beta)
$$

and give estimates for these three terms separately. These terms come from the expression of $d_{n}(\alpha, \beta)$ given in Proposition 4.1. The first term arises by summing the sequence of constants $C\left(\alpha_{m}, n(m)\right)$. The second comes from summation of the terms $S(n, \alpha, \beta)$. The last term arises by adding the different $\bar{\beta}^{m}$ that we encounter when $b_{m}>0$. In the appendix we give the proof of the following estimates 
Proposition 4.3. Let $n$ be an integer such that ord $(n-1)=N$, then for all $\alpha=\left[a_{1}, a_{2}, \ldots\right]$ and all $\beta$, the following hold:

(i) $|\mathcal{C}(n, \alpha, \beta)|<N$;

(ii) $|\mathcal{B}(n, \alpha, \beta)|<N$;

(iii) $|\mathcal{S}(n, \alpha, \beta)| \leq \sum_{m=1}^{N+1}\left(1+\frac{a_{m}}{4}\right)$.

We now compute the sum $S_{n}(\alpha, \beta)$ for a given $\alpha$. Let

$$
S_{n}(\alpha, \beta)=S_{n}(\alpha)+R_{n}(\alpha, \beta)
$$

where the term $R_{n}(\alpha, \beta)$ accounts for the times that $s(\{r \alpha\}) \neq s(\{r \alpha+\beta\})$. We first remark that for all $\beta \in\left[0, \frac{1}{2}\right)$ it holds

$$
S_{n}\left(\alpha, \beta+\frac{1}{2}\right)=-S_{n}(\alpha, \beta)
$$

hence it suffices to study $R_{n}(\alpha, \beta)$ in the case $\beta<\frac{1}{2}$.

Theorem 4.4. For all $\beta \in\left[0, \frac{1}{2}\right)$,

$$
S_{n}(\alpha, \beta)=S_{n}(\alpha)+R_{n}(\alpha, \beta)
$$

where the term $R_{n}(\alpha, \beta)$ can be written as

$$
R_{n-1}(\alpha, \beta)=2\left[d_{n}\left(\alpha, \frac{1}{2}-\beta\right)-d_{n}(\alpha, 1-\beta)-d_{n}\left(\alpha, \frac{1}{2}\right)\right]
$$

Proof. If we denote

$$
\begin{gathered}
P_{n}:=\left\{0 \leq r \leq n-1: \frac{1}{2}<\{r \alpha\}<1, \quad 1<\beta+\{r \alpha\}<\frac{3}{2}\right\} \\
M_{n}:=\left\{0 \leq r \leq n-1:\{r \alpha\}<\frac{1}{2}, \quad \frac{1}{2}<\beta+\{r \alpha\}<1\right\}
\end{gathered}
$$

then

$$
R_{n-1}(\alpha, \beta)=2\left(\operatorname{card}\left(P_{n}\right)-\operatorname{card}\left(M_{n}\right)\right)
$$

Introducing the notations

$$
\begin{gathered}
A_{a}^{b}:=\{0 \leq r \leq n-1: a<\{r \alpha\}<b\} \\
B_{a}^{b}:=\{0 \leq r \leq n-1: a<\beta+\{r \alpha\}<b\}
\end{gathered}
$$


we have

$$
\begin{gathered}
P_{n}=B_{1}^{3 / 2} \cap A_{1 / 2}^{1} \\
M_{n}=B_{1 / 2}^{1} \cap A_{0}^{1 / 2}=\left(B_{1 / 2}^{\infty} \cap A_{0}^{1 / 2}\right) \backslash\left(B_{1}^{\infty} \cap A_{0}^{1 / 2}\right)
\end{gathered}
$$

Now, since $0 \leq \beta<\frac{1}{2}$, it is easy to obtain

$$
\begin{gathered}
B_{1}^{3 / 2} \cap A_{1 / 2}^{1}=B_{1}^{3 / 2} \\
B_{1}^{\infty} \cap A_{0}^{1 / 2}=\emptyset
\end{gathered}
$$

hence

$$
\begin{gathered}
P_{n}=B_{1}^{3 / 2}=A_{(1-\beta)}^{1}=A_{0}^{1} \backslash A_{0}^{(1-\beta)} \\
M_{n}=B_{1 / 2}^{\infty} \cap A_{0}^{1 / 2}=A_{0}^{1 / 2} \backslash A_{0}^{(1 / 2-\beta)}
\end{gathered}
$$

The thesis now follows by writing

$$
\operatorname{card}\left(A_{a}^{b}\right)=\sum_{r=0}^{n-1} \chi_{(a, b)}(\{r \alpha\})
$$

and using the definition of $d_{n}(\alpha, \beta)$ in (4.1).

Putting together Proposition 4.3 and Theorem 4.4, one gets immediately

Corollary 4.5. For all irrational $\alpha=\left[a_{1}, a_{2}, \ldots\right]$ it holds

$$
\sup _{\beta}\left|S_{n}(\alpha, \beta)\right| \leq\left|S_{n}(\alpha)\right|+6 \sum_{m=1}^{N}\left(3+\frac{a_{m}}{4}\right)
$$

where $\operatorname{ord}(n)=N$.

\subsection{Applications}

We now give some applications of the estimates of Proposition 4.3 ,

We first consider the speed of convergence in the Birkhoff Ergodic Theorem. As stated in the Introduction, the Lebesgue measure is invariant and ergodic for the irrational translations on the unit circle. Hence for the rotation $T_{\alpha}(\beta):=\{\alpha+\beta\}$, the Birkhoff Ergodic Theorem implies that

$$
\lim _{n \rightarrow \infty} \frac{1}{n} \sum_{k=0}^{n-1} \chi_{I}(\{k \alpha+\beta\})=|I|:=\int_{0}^{1} \chi_{I}(x) d x
$$

for any interval $I \subset[0,1]$. We prove that 
Theorem 4.6. For any interval $I \subset[0,1]$ it holds

$$
\sum_{k=0}^{n-1} \chi_{I}(\{k \alpha+\beta\})-n|I|=d_{n}(\alpha,\{\delta-\beta\})-d_{n}(\alpha,\{\gamma-\beta\})
$$

from which it follows that

$$
\left|\sum_{k=0}^{n-1} \chi_{I}(\{k \alpha+\beta\})-n\right| I|| \leq 2 \sum_{m=1}^{N}\left(3+\frac{a_{m}}{4}\right)
$$

where $\alpha=\left[a_{1}, a_{2}, \ldots\right]$ and $\operatorname{ord}(n-1)=N$.

Proof. Let $I=[\gamma, \delta]$. Then we can write

$$
\sum_{k=0}^{n-1} \chi_{I}(\{k \alpha+\beta\})=\sum_{k=0}^{n-1} \chi_{[\gamma-\beta, \delta-\beta]}(\{k \alpha\})
$$

By using equation (4.1), we obtain (4.8). The estimate (4.9) follows by Proposition 4.3 ,

One can easily generalize (4.8) to bounded variation functions, for which the analogous of estimate (4.9) is the Denjoy-Koksma inequality (see [KN]). Indeed estimate (4.9) can be considered a particular case of the DenjoyKoksma inequality.

As stated at the beginning of Section 4, the term $d_{n}(\alpha, \beta)$ is related to the discrepancy $D_{n}^{*}(\alpha)$ of the sequence $(n \alpha)$ by equation (4.2). Some classical results are known for the discrepancy of a general sequence, and in particular for the sequence $(n \alpha)$ according to the arithmetical properties of $\alpha$ (see $[\mathrm{KN}]$ ). For more recent sharp results we refer to $[\mathrm{Sc}$ and $[\mathrm{P}]$. We briefly show below how to get similar results to those in $[\mathrm{Sc}$ and $[\mathrm{P}$ directly by our approach. Proofs can be found in the appendix.

From Proposition 4.3 one gets immediately

$$
n D_{n}^{*}(\alpha)=\sup _{\beta}\left|d_{n}(\alpha, \beta)\right| \leq 1+3 N+\frac{1}{4} \sum_{m=1}^{N+1} a_{m}
$$

where $N=\operatorname{ord}(n-1)$. This bound is of the same order of results in $[\mathrm{P}]$. We will show that this is the best possible estimate for the general case. But first we shall obtain some lower bounds. 
Proposition 4.7. For all irrational $\alpha$ there exist a number $\beta$ and two infinite subsequences $\left(n_{k}\right)$ and $\left(n_{h}\right)$ such that

$$
\sup _{\beta}\left|\mathcal{S}\left(n_{k}, \alpha, \beta\right)\right| \geq \sum_{\substack{m=1 \\ a_{2 m-1} \geq 3}}^{\frac{N_{k}}{2}+1} \frac{a_{2 m-1}-2}{4}
$$

where $N_{k}=\operatorname{ord}\left(n_{k}\right)$ and

$$
\sup _{\beta}\left|\mathcal{S}\left(n_{h}, \alpha, \beta\right)\right| \geq \sum_{\substack{m=2 \\ a_{2 m} \geq 3}}^{\frac{N_{h}+1}{2}} \frac{a_{2 m}-2}{4}
$$

where $N_{h}=\operatorname{ord}\left(n_{h}\right)$.

Theorem 4.8. Let $\alpha$ have unbounded partial quotients $\left(a_{k}\right)$ and denote

$$
\begin{gathered}
\ell_{e}=\liminf _{k \rightarrow \infty} \frac{\sum_{m=1 \text { even }}^{k} a_{m}}{\sum_{m=1}^{k} a_{m}} \\
\ell_{o}=\liminf _{k \rightarrow \infty} \frac{\sum_{m=1 \text { odd }}^{k} a_{m}}{\sum_{m=1}^{k} a_{m}}
\end{gathered}
$$

If $\left(\ell_{e}^{2}+\ell_{o}^{2}\right)>0$ and

$$
\limsup _{k \rightarrow \infty} \frac{k}{\sum_{m=1}^{k} a_{m}}=0
$$

then

$$
\frac{1}{4} \max \left\{\ell_{e}, \ell_{o}\right\} \leq \limsup _{n \rightarrow \infty} \frac{n D_{n}^{*}(\alpha)}{\sum_{m=1}^{\operatorname{ord}(n-1)+1} a_{m}} \leq \frac{1}{4}
$$

ExAMPLE. The conditions of Theorem 4.8 are satisfied by

$$
\alpha=e-2=[1,2,1,1,4,1,1,6,1,1,8,1,1,10,1,1,12, \ldots]
$$

indeed $\ell_{e}=\ell_{o}=\frac{1}{2}$ and $\sum_{m=1}^{k} a_{m} \sim \frac{1}{9} k^{2}$.

In fact estimate (4.12) is the best possible in general, as can be shown by choosing for example

$$
\alpha:=[1,2,1,3,1,4,1,5, \ldots, 1, n, \ldots]
$$


for which $\ell_{e}=1, \ell_{o}=0$ and $\sum_{m=1}^{k} a_{m} \sim \frac{1}{8} k^{2} . \diamond$

We finish with few more remarks. First of all, we remark that by Proposition 2.4 one can easily prove the well known result that for a given $\alpha \in(0,1)$, if $\beta \in \mathbb{Z}+\alpha \mathbb{Z}$ then $d_{n}(\alpha, \beta)$ is bounded for all $n \geq 0$.

Finally, we dwell upon relations between the discrepancy and the sums $S_{n}(\alpha)$. To start with, let us notice that by definition $S_{n-1}(\alpha)=2 d_{n}\left(\alpha, \frac{1}{2}\right)$, so that for any given function $F(n) \nearrow \infty$ we have

$$
\limsup _{n \rightarrow \infty} \frac{n D_{n}^{*}(\alpha)}{F(n)} \geq \limsup _{n \rightarrow \infty} \frac{\left|S_{n-1}(\alpha)\right|}{2 F(n)}
$$

The analogous relation for the infimum limit is not interesting since for all $\alpha$ it holds $\left|S_{q_{k}}(\alpha)\right| \leq 2$ for all denominators $q_{k}$ (see [I] ).

However, in some cases one could get an equality in (4.13). Let us consider the case $\alpha=\sqrt{2}-1=[2,2,2, \ldots]$. We find from Theorem 3.2

$$
\limsup _{n \rightarrow \infty} \frac{n D_{n}^{*}(\sqrt{2}-1)}{\log n} \geq \limsup _{n \rightarrow \infty} \frac{\operatorname{ord}(n-1)}{4 \log n} \geq \frac{1}{4 \log (\sqrt{2}+1)}
$$

This relation is in fact an equality, as proved in [DS], and therefore

$$
\limsup _{n \rightarrow \infty} \frac{S_{n}(\sqrt{2}-1)}{2 \log n}=\limsup _{n \rightarrow \infty} \frac{n D_{n}^{*}(\sqrt{2}-1)}{\log n}
$$

The same is shown in $\left[\mathrm{A}\right.$ for $\alpha=\frac{\sqrt{3}-1}{2}=[2,1,2,1, \ldots]$. Moreover the author exhibits some other couples $(\alpha, \beta)$ for which a similar relation holds. He then conjectures that a similar relation holds for all couples $(\alpha, \beta)$ with $\alpha$ a quadratic irrational and $\beta \in \mathbb{Q}(\alpha)$ but $\beta \notin \mathbb{Z}+\alpha \mathbb{Z}$. We show here that it is not the case.

Corollary 4.9. Let $\alpha$ be a quadratic irrational in $(0,1)$ with partial quotients $\left(a_{m}\right)$ verifying $a_{2 i-1}=2$ and $a_{2 i}=2 k$ for all $i \geq 1$ and a fixed $k>2$. Then

$$
\nu^{*}(\alpha)>\limsup _{n \rightarrow \infty} \frac{\left|d_{n}\left(\alpha, \frac{1}{2}\right)\right|}{\log n}
$$

Proof. Applying the result of $[\mathrm{Sc}]$ mentioned above it follows that

$$
\limsup _{n \rightarrow \infty} \frac{n D_{n}^{*}(\alpha)}{\log n}=\frac{1}{4} \max \left\{\limsup _{N \rightarrow \infty} \frac{k N}{\log q_{N}}, \limsup _{N \rightarrow \infty} \frac{N}{\log q_{N}}\right\}
$$

However from Theorem 3.2 and equation (4.13), it follows that if $N=\operatorname{ord}(n)$ then

$$
\limsup _{n \rightarrow \infty} \frac{\left|d_{n}\left(\alpha, \frac{1}{2}\right)\right|}{\log n} \leq \limsup _{n \rightarrow \infty} \frac{N}{2 \log n} \leq \limsup _{n \rightarrow \infty} \frac{N}{2 \log q_{N}}
$$

Hence, if $k>2$, the thesis is proved. 


\section{Appendix}

Proof of Proposition 4.3. Part (i) is an easy consequence of Lemma 4.2, which implies that for all $\beta$

$$
|\mathcal{C}(n, \alpha, \beta)| \leq \sum_{m=0}^{N-1} a_{1}^{(m)} \alpha_{m}
$$

hence the thesis, since $a_{1}^{(m)} \alpha_{m}<1$. The same holds true if any of the terms of the sum is of the type $\bar{a}_{1}^{(m)} \bar{\alpha}_{m}$.

The proof of part (ii) is even more immediate, since

$$
\mathcal{B}(n, \alpha, \beta) \leq \sum_{m=1}^{N} \bar{\beta}^{m}
$$

with $\beta^{m}<1$ for all $m$. Note that having an equality in the previous relation would mean that each step of the renormalization procedure is done using $\bar{\alpha}_{m}$ and $\bar{\beta}^{m}$.

To study the behaviour of $\mathcal{S}(n, \alpha, \beta)$ let us start writing $n-1=r_{k_{j}}+R_{1} q_{1}+$ $R_{0}$ as usual. It is then easy to realise that $S(n, \alpha, \beta)$ can assume only a finite number of values. In particular, for each $\beta$, a short calculation shows that

$$
\left(b_{0}+1\right)(1-\beta) \geq S(n, \alpha, \beta) \geq \begin{cases}b_{0}-a_{1} \beta-\beta, & \text { if } b_{1}=0, \\ b_{0}-a_{1} \beta, & \text { if } b_{1}>0,\end{cases}
$$

for all $n \in\left[r_{k_{j}}, r_{k_{j+1}}-1\right]$. A similar result holds for $S^{c}(n, \alpha, \beta)$, namely

$$
\begin{cases}b_{0}-a_{1} \beta+1-\beta \geq S^{c}(n, \alpha, \beta) \geq-\beta\left(a_{1}+1-b_{0}\right), & \text { if } b_{1}=0, \\ b_{0}-a_{1} \beta+1 \geq S^{c}(n, \alpha, \beta) \geq-\beta\left(a_{1}-b_{0}\right), & \text { if } b_{1}>0 .\end{cases}
$$

Let us first examine $|S(n, \alpha, \beta)|$. We have that if $b_{1}=0$ then $\left(b_{0}-a_{1} \beta-\beta\right) \geq$ -1 , whereas if $b_{1}>0$ then $\left(b_{0}-a_{1} \beta\right) \geq-a_{1} \alpha$. Moreover, maximising $\left(b_{0}+1\right)(1-\beta)$ on $(0,1)$ yields for all $\alpha$ and $\beta$

$$
|S(n, \alpha, \beta)| \leq \begin{cases}\left(\frac{a_{1}}{2}+1\right)\left(1-\frac{a_{1}}{2} \alpha\right) \leq 1+\frac{a_{1}}{4}, & \text { if } a_{1} \text { is even }, \\ \frac{a_{1}+1}{2}\left(1-\frac{a_{1}-1}{2} \alpha\right) \leq 1+\frac{a_{1}-1}{4}, & \text { if } a_{1} \text { is odd }\end{cases}
$$

For $\left|S^{c}(n, \alpha, \beta)\right|$, if $b_{1}=0$ then $\left(b_{0}-a_{1} \beta+1-\beta\right) \leq 1$, if $b_{1}>0$ then $\left(b_{0}-a_{1} \beta+1\right) \leq a_{1} \alpha$. To maximise $\left|S^{c}(n, \alpha, \beta)\right|$ we have to consider separately the cases $b_{1}=0$ and $b_{1}>0$. 
If $b_{1}=0$, then maximising $\beta\left(a_{1}+1-b_{0}\right)$, for all $\alpha$ and $\beta$ we get

$$
\beta\left(a_{1}+1-b_{0}\right) \leq \begin{cases}\left(\frac{a_{1}}{2}+1\right)^{2} \alpha \leq 1+\frac{a_{1}+a_{2}}{4}, & \text { if } a_{1} \text { is even } \\ \frac{\left(a_{1}+1\right)\left(a_{1}+3\right)}{4} \alpha \leq 1+\frac{a_{1}+a_{2}}{4}, & \text { if } a_{1} \text { is odd }\end{cases}
$$

But if $b_{1}=0$, in the next step of the renormalization, the partial quotient $a_{2}$ will not appear (see the scheme (4.5)).

If $b_{1}>0$, we have instead to maximise $\beta\left(a_{1}-b_{0}\right)$. For all $\alpha$ and $\beta$ it holds

$$
\beta\left(a_{1}-b_{0}\right) \leq \begin{cases}\left(\frac{a_{1}}{2}+1\right) \frac{a_{1}}{2} \alpha \leq 1+\frac{a_{1}}{4}, & \text { if } a_{1} \text { is even } \\ \left(\frac{a_{1}+1}{2}\right)^{2} \alpha \leq 1+\frac{a_{1}}{4}, & \text { if } a_{1} \text { is odd }\end{cases}
$$

Applying these inequalities to each renormalization step yields

$$
\mathcal{S}(n, \alpha, \beta) \leq \sum_{m=0}^{N}\left(1+\frac{a_{1}^{(m)}}{4}\right)
$$

where we are using $\bar{a}_{1}^{(m)}=a_{1}^{(m)}-1<a_{1}^{(m)}$. The thesis of part (iii) follows using $a_{1}^{(m)}=a_{m+1}$, where $\left(a_{k}\right)$ are the partial quotients of $\alpha$.

Proof of Proposition 4.7. We need to show the existence of a $\beta$ such that $d_{n}(\alpha, \beta)$ has the form we look for. Let $\beta$ satisfy $b_{2 k+1}=0$ for all $k \geq 0$, then following the scheme (4.5) one works with couples which are all of the form $\left(\alpha_{2 m}, \beta^{2 m-1}\right)$, for $m \geq 0$ (we denote $\beta \equiv \beta^{-1}$ ), and there are no inversions. Hence one has $\mathcal{S}(n, \alpha, \beta)=\sum_{m=0}^{\frac{\operatorname{ord}(n-1)}{2}+1} S\left(n(m), \alpha_{2 m}, \beta^{2 m-1}\right)$, where $n(m)$ denotes the number of iterates at each step, that is $n(0)=n$, $n(1)=j(n-1)+1$, and in general $n(m)=j(n(m-1)-1)+1$. We now remark that for all integers of the form $r_{k_{j}}+R_{1} q_{1}$ there exists $\bar{R}_{0}$ such that if $n-1=r_{k_{j}}+R_{1} q_{1}+\bar{R}_{0}$ then $S\left(n(m), \alpha_{2 m}, \beta^{2 m-1}\right)=b_{0}^{(2 m)}\left(1-\beta^{2 m-1}\right)$ for all $m$, and since this choice depends only on $R_{0}$, and changing $R_{0}$ does not change $j(n-1)$ (see equation (3.3)), we can choose a sequence $n_{k}$ such that at each renormalization step the term $S$ has the chosen value. Moreover, we can show that if $a_{1}^{(2 m)} \geq 3$ then $b_{0}^{(2 m)}\left(1-\beta^{2 m-1}\right)$ is bigger than $\frac{a_{1}^{(2 m)}-2}{4}$ for all $m \geq 0$ if $b_{0}^{(2 m)}=\frac{a_{1}^{(2 m)}}{2}$ or $b_{0}^{(2 m)}=\frac{a_{1}^{(2 m)}-1}{2}$, according to whether $a_{1}^{(2 m)}$ is even or odd, respectively. If $a_{1}^{(2 m)}<3$, we can choose $\bar{R}_{0}$ such that $S \geq 0$. The first part follows.

For the second part of the proof, we just change a little bit the argument, choosing $\beta$ such that $b_{1}>0$ and $b_{2 k}=0$ for all $k \geq 1$. Then by the 
scheme (4.5), we use all the couples $\left(\alpha_{2 m+1}, \beta^{2 m}\right)$ for all $m \geq 1$, and they are all inverted, since there is only one inversion at the beginning. Hence $\mathcal{S}(n, \alpha, \beta)=S(n, \alpha, \beta)+\sum_{m=1}^{\frac{\operatorname{ord}(n-1)}{2}+1} S^{c}\left(n(m), \alpha_{2 m+1}, \beta^{2 m}\right)$. As above, we can choose a subsequence $n_{h}$ such that for all $m \geq 1$ it holds

$$
S^{c}\left(n(m), \alpha_{2 m+1}, \beta^{2 m}\right)=-\beta^{2 m}\left(a_{1}^{(2 m+1)}-b_{0}^{(2 m+1)}-1\right) \leq-\frac{a_{1}^{(2 m+1)}-2}{4}
$$

by choosing $b_{0}^{(2 m+1)}=\frac{a_{1}^{(2 m+1)}}{2}$ or $b_{0}^{(2 m+1)}=\frac{a_{1}^{(2 m+1)}-1}{2}$, according to whether $a_{1}^{(2 m+1)}$ is even or odd respectively, if $a_{1}^{(2 m)} \geq 3$. Otherwise, just choose $S^{c} \leq 0$.

Proof of Theorem 4.8. The upper bound follows straightforwardly from (4.10). Moreover, from Propositions 4.3 and 4.7, we obtain that if $\left(\ell_{e}^{2}+\ell_{o}^{2}\right)>$ 0 then there exists a subsequence $\left(n_{h}\right)$ such that, denoting $N_{h}=\operatorname{ord}\left(n_{h}\right)$, for all $\epsilon>0$ there exists $\bar{h}$ such that for all $h>\bar{h}$

$$
n_{h} D_{n_{h}}^{*} \geq \sup _{\beta}\left|\mathcal{S}\left(n_{h}, \alpha, \beta\right)\right|-2 N_{h} \geq \frac{\max \left\{\ell_{e}, \ell_{o}\right\}-\epsilon}{4} \sum_{m=1}^{N_{h}+1} a_{m}-3 N_{h}
$$

where we have used the relation

$$
\sum_{\substack{m=1 \\ a_{m} \geq 3}}^{k} a_{m} \geq \sum_{m=1}^{k} a_{m}-2 k
$$

for all $k \geq 1$, which holds also when the sum is restricted to even or odd indexes. From this it follows

$$
\limsup _{n \rightarrow \infty} \frac{n D_{n}^{*}(\alpha)}{\sum_{m=1}^{\operatorname{ord}(n-1)+1} a_{m}} \geq \frac{\max \left\{\ell_{e}, \ell_{o}\right\}-\epsilon}{4}
$$

for all $\epsilon>0$.

\section{References}

[A] B.Adamczewski, Répartition des suites $(n \alpha)_{n \in \mathbb{N}}$ et substitutions, Acta Arith. 112 (2004), 1-22

[B] C.Bonanno, Limit laws of rotations, in preparation 
[B2] C.Bonanno, S.Galatolo, S.Isola, Recurrence and algorithmic information, Nonlinearity 17 (2004), 1057-1074

[CD] J.-R.Chazottes, F.Durand, Local rates of Poincaré recurrence for rotations and weak mixing, Discrete Contin. Dyn. Syst. 12 (2005), 175-183

[CF] Z.Coelho, E.de Faria, Limit laws of entrance times for homeomorphisms of the circle, Israel J. Math. 93 (1996), 93-112

[DS] Y.Dupain, V.T.Sós, On the discrepancy of $\{n \alpha\}$ sequences, in: "Topics in Classical Number Theory. Vol. I", G.Halász (ed.), Colloquia Mathematica Societatis János Bolyai 34, North Holland, 1984, pp. 355-387

[I] S.Isola, Dispersion properties of ergodic translations, Int. J. Math. Math. Sci., Vol. 2006, Art.20568

[K] D.H.Kim, The recurrence time for irrational rotations, Osaka J. Math. 43 (2006), 351-364

[KS] D.H.Kim, B.K.Seo, The waiting time for irrational rotations, Nonlinearity 16 (2003), 1861-1868

[KN] L.Kuipers, H.Niederreiter, "Uniform Distribution of Sequences", John Wiley and Sons, New York, 1974

[L] O.E.Lanford, Renormalization group methods for circle mappings, in "Nonlinear Evolution and Chaotic Phenomena", NATO Adv. Sci. Inst. Ser. B: Phys., 176, Plenum, New York, 1988, pp. 25-36

[P] C.Pinner, On sums of fractional parts $\{n \alpha+\gamma\}$, J. Number Theory 65 (1997), 48-73

[PF] N.Pytheas Fogg (V.Berthé, S.Ferenczi, C.Mauduit, A.Siegel eds.), "Subsitutions in Dynamics, Arithmetics and Combinatorics", Lecture Notes in Mathematics 1794, Springer Verlag, Berlin, 2002

[Sc] J.Schoissengeier, The discrepancy of $\{n \alpha\}$, Math. Ann. 296 (1993), $529-545$

[So] V.T.Sós, On the discrepancy of the sequence $\{n \alpha\}$, Coll. Math. Soc. J. Bolyai 13 (1976), "Topics in Number Theory", ed. P. Turán 